\title{
A REVIEW ON DEVELOPMENT OF BIORELEVANT DISSOLUTION MEDIUM
}

\author{
*Bhagat Nitin B. ${ }^{1}$, Yadav Adhikrao.V. ${ }^{1}$, Mali Sachin.S. ${ }^{2}$, Khutale Rohan A. ${ }^{1}$, Hajare Ashok A. ${ }^{2}$, \\ Salunkhe Sachin S. ${ }^{2}$, Nadaf Sameer J. ${ }^{2}$ \\ ${ }^{1}$ Department of Pharmaceutics, Gourishankar Institute of Pharmaceutical Education and Research, Limb Satara, \\ Maharashtra, India \\ ${ }^{2}$ Department of Pharmaceutical Technology, Bharati Vidyapeeth College of Pharmacy, Near Chitranagari, Kolhapur, \\ Maharashtra, India \\ *Corresponding Author's E-mail: sachinmali143@gmail.com
}

\begin{abstract}
Dissolution testing is a valuable tool that provides key information about bioavailability or bioequivalency as well as batch to batch consistency of drug. Since the number of poorly soluble drug is increasing, the selection of adequate dissolution test for these becomes more and more important. Biorelevant is a term, used to describe a medium that has same relevance to the in vivo dissolution condition for the compound. The development of biorelevant dissolution medium includes simulation of gastrointestinal condition, hydrodynamic characteristics, and physicochemical parameters of drug, prediction of plasma profile and lastly the development of IVIVC. Biorelevant dissolution testing designed with appropriate simulated media and hydrodynamics are useful from the early stages of drug discovery and development for identifying the biopharmaceutical performance of compound (i.e. solubility problems, food effects, precipitation in the small intestine) through the later stages of development to assist in formulation strategies and the establishment of IVIVC that will lead to reduction of the number of animal experimentation, bioavailability and bioequivalence studies. The aim of this review article is to provide comprehensive information on the steps that should be considered during developing biorelevant dissolution medium for poorly soluble drugs and the composition of biorelevant dissolution medium to predict in vivo performance more accurately than the compendia dissolution medium.

Keywords: Biorelevant, Simulated gastrointestinal condition, hydrodynamic, IVIVC etc.
\end{abstract}

\section{INTRODUCTION}

Dissolution of drugs from solid dosage forms is a key parameter in assessing the product quality and uniformity at the formulation stage as well as throughout the shelflife of the product ${ }^{1}$. In case of lipophilic drugs dissolution rate can be the rate-limiting step in the in vivo absorption process and hence the dissolution medium is a critical component of the test that can cause problems ${ }^{2,3}$. Therefore, dissolution method should be discriminative, reproducible, scientifically justifiable and more importantly biorelevant ${ }^{4}$. This clinical relevance of dissolution testing can be achieved in the context of Quality by Design derived from a specific case study for a BCS 2 compound $^{5}$.

Approaches usually used in the design of a dissolution media for poorly water soluble drugs include: ${ }^{(6)}$

1) Bringing about drug solubility by increasing the volume of the aqueous sink or removing the dissolved drug.

2) Solubilization of drug by cosolvents up to $40 \%$ by addition of anionic or non-anionic surfactants to dissolution medium in post micellar concentration.

3) Alteration of $\mathrm{pH}$ to enhance the solubility of insoluble drug molecule.

Surfactant solutions are often proposed as dissolution media for drugs characterized by low water solubility. Generally, aqueous solutions of such surfactants may simulate the physiological environment more accurately rather than using adsorbents or hydroalcoholic and aliphatic media ${ }^{7}$. However, Tang and coworkers showed that for a low solubility drug, increase in solubility by addition of surfactants to meet sink conditions (based on bulk drug solubility data) may not always produce biorelevant results ${ }^{8}$.

Aqueous buffers can be used to reflect typical $\mathrm{pH}$ conditions in the stomach or small intestine, but do not represent other key aspects of the composition of the GI contents (e.g., Osmolality, ionic strength, viscosity, surface tension) that can be relevant to drug release from the dosage form to be tested. In particular, they cannot be used to simulate the influence of food ingestion on drug release.

Normal adult diet contains about $150 \mathrm{gm}$ of lipids, $95 \%$ of which are long-chain triglycerides and 4-8gm of phospholipids mainly composed of lecithin ${ }^{9}$. In fed state bioavailability of drug can be increased when there will be change in GIT environment such as ${ }^{10}$

1) Prolonged gastric emptying and decrease in intestinal motility increasing the time available for Solubilization.

2) Increased dissolution rate and Solubilization of drug substances in mixed micells due to simulation of pancreatic secretion of bile salts and lipase.

3) Protection from gastric/luminal degradation, due to protection in lipids. 
4) Increased lymphatic transport, thus avoiding first pass metabolism.

Specifically in the case of poorly soluble compounds, it is often observed that the in vivo fraction absorbed increases when the drug is given with a meal. Thus, in order to simulate the effects of food on dissolution in the GI tract, it is equally important to develop representative dissolution tests for both the fasted and fed states ${ }^{11}$.

This article mainly focuses on composition of Biorelevant dissolution medium for poorly water soluble drug with the necessary steps that need to be considered for development of Biorelevant Dissolution Medium.

\section{Bio-relevant Dissolution Media}

Compendial dissolution media often fail to yield IVIVC's for class 2 drugs because relevant physiological parameters are not taken into account. A suitable in vitro model should include a medium that mimics as much as possible the GIT contents after food intake 12, 13 . Biorelevant in vitro dissolution testing is useful for qualitative forecasting of formulation and food effects on the dissolution and availability of orally administered drugs ${ }^{14,15}$. These biorelevant media can be used to assess the performance of different formulations for poorly water soluble compounds. Biorelevant media have been successfully applied over the past decade to obtain IVIVCs ${ }^{16}, 17,{ }^{18}$. Two bio-relevant dissolution media simulating conditions in the proximal small intestine FaSSIF and FeSSIF were proposed in 1998.

Bio-relevant dissolution methods, combined with permeability measurements and computational simulations, were used to predict the oral absorption of drug ${ }^{19}$. Due to their complex composition, these media are expensive and need to be prepared on the day of the experiment ${ }^{20}$.

Before the development of biorelevant dissolution medium the following steps should be considered

1) Fluid composition in the GIT

2) Hydrodynamics in the GIT

3) API/formulation properties

4) Prediction of plasma profile

5) Development of IVIVCs

\section{1) Fluid composition of GIT}

The features of GI fluid are altered in fasted and fed condition and they affect the dissolution. Several physiochemical and physiological properties of GI fluids such as $\mathrm{pH}$, buffer capacity, bile component concentration and state of aggregation and enzyme activity can greatly influence the drug dissolution process 21, 22, 23. For simulation of GI fluid the composition of GI fluid plays important role because upon simulating biological environment after a convenient alternative could facilitate routine and experimental in vitro dissolution work ${ }^{(24)}$. Several physiologically based models for GI transit and absorption have been developed recently ${ }^{25}$.

\section{Stomach:}

Motility in the stomach and small bowel is organized into two basic motor patterns fasting and fed. Fasting motor pattern is characterized by cyclic repetition of periods of quiescence altering with periods of contractile activity. Fed motor pattern is characterized by irregular but persistant phasic contractile activity. It develops almost immediately after ingestion of food and replaces the fasting pattern at whatever point in the interdigestive cycle the meal is eaten ${ }^{26}$. Under fasting condition $\mathrm{pH}$ of healthy human stomach is acidic, ranging between 1 and 3 . Fluid volume in the stomach would initially be around $300 \mathrm{ml}$ in fasted state and $500 \mathrm{ml}$ or more in the fed state. The problem while carrying out the dissolution test in FeSSGF is that the medium contains milk, which cannot be filtered using filters with a pore size in the range of 20-500 nm.

\section{Intestine:}

Motility of intestine comprises of intraluminal flow, motion of the wall that induce the flow and systems that regulate the wall motions ${ }^{26}$. Fluid volume in small intestine is of $200 \mathrm{ml}$ in fasted state and $1 \mathrm{~L}$ in the fed state ${ }^{27}$. It has been found that the bioavailability of poorly soluble drug can be markedly enhanced by meal intake and its related changes in GI tract physiology such as secretions, digestion processes and motility ${ }^{28}$. The human intestinal fluid contains bile salts, phospholipids, monoglycerides, free fatty acids and cholesterol. The increased solubility in FeSSIF-V2 can be explained by the formation of solubilizing micelles from bile salts, lecithin, GMO and sodium oleate ${ }^{29}$.

Vertzoni et al have proposed that the surface tension could be lowered appropriately with combination of pepsin and very low concentration of bile salt. Bile salts and lecithin can increase the wetting process for the lipophilic drugs and solubilize the drug into the micelles formed by bile salts and lecithin. Composition of bile salts in human shown in table $1^{30}$.

Table 1: Composition of bile salts in human

\begin{tabular}{|l|c|}
\hline Conjugated bile acids & $\begin{array}{l}\text { Percentage in } \\
\text { bile salts }\end{array}$ \\
\hline Cholyglycine & 24 \\
\hline Chenglycine & 24 \\
\hline Deoxycholyglycine & 16 \\
\hline Cholyltaurine & 12 \\
\hline chenyltaurine & 12 \\
\hline deoxycholytaurine & 8 \\
\hline Sulfolithocholylglycine & 3 \\
\hline Sulfolithocholytaurine & 1 \\
\hline Lithocholyglycine & 0.7 \\
\hline Lithocholyltaurine & 0.3 \\
\hline
\end{tabular}

\section{Colon:}

Unlike the motility of the stomach and small intestine which is characterized by the cyclic appearance of the migrating motor complex under fasted conditions, colonic motility is rather limited and in progress due to its inaccessibility and regional differences in structure and function. 
The composition of biorelevant media which are proposed by dissolution scientist in fasted and fed state for stomach, intestine and colon are shown in table from $2-6$

Table 2: Composition of the Media to Simulate Gastric Contents in the Fasted State

\begin{tabular}{|l|l|l|l|}
\hline Gastric Contents & SGF $_{\text {SLS }}$ & SGF $_{\text {Triton }}$ & FaSSGF \\
\hline Sodium lauryl sulfate $(\% \mathrm{w} / \mathrm{v})$ & $0.25 / 0.05$ & - & - \\
\hline Triton X 100 $(\% \mathrm{w} / \mathrm{v})$ & - & 0.1 & - \\
\hline Pepsin $(\mathrm{mg} / \mathrm{ml})$ & - & - & 0.1 \\
\hline NaTc $(\mu \mathrm{m})$ & - & - & 80 \\
\hline Lecithin $(\mu \mathrm{m})$ & - & - & 20 \\
\hline $\mathrm{NaCl}$ & 34.2 & 34.2 & 34.2 \\
\hline $\mathrm{pH}$ & 1.2 & 1.2 & 1.6 \\
\hline Surface Tension $(\mathrm{mN} / \mathrm{m})$ & 33.7 & 32.0 & 42.6 \\
\hline Osmolality $(\mathrm{mOsml} / \mathrm{Kg})$ & $180 \pm 3.6$ & $157.7 \pm 2.9$ & $120.7 \pm 2.5$ \\
\hline
\end{tabular}

Table 3: Composition of the Media to Simulate Gastric contents in the Fed State

\begin{tabular}{|l|l|l|l|}
\hline Gastric Contents & Early & Middle & Late \\
\hline Sodium chloride $(\mathrm{mM})$ & 148 & 237.02 & 122.6 \\
\hline Acetic acid (mM) & - & 17.12 & - \\
\hline Sodium acetate( mM) & - & 29.75 & - \\
\hline Ortho-phosphoric acid (mM) & - & - & 5.5 \\
\hline Sodium dihydrogen phosphate $(\mathrm{mM})$ & - & - & 32 \\
\hline Milk: buffer & $1: 0$ & $1: 1$ & $1: 3$ \\
\hline Hydrochloric acid/sodium hydroxide & qs pH 6.4 & qs pH 5 & qs pH 3 \\
\hline pH & 6.4 & 5 & 3 \\
\hline Osmolality $(\mathrm{mOsmol} / \mathrm{Kg})$ & $559 \pm 10$ & $400 \pm 10$ & $300 \pm 10$ \\
\hline Buffer capacity $(\mathrm{mml} / \mathrm{pH})$ & 21.33 & 25 & 25 \\
\hline
\end{tabular}

Table 4: Composition of the Media to Simulate the Contents of the Small Intestine in the Fasted State

\begin{tabular}{|l|l|l|}
\hline Contents of the Small Intestine & FaSSIF & FaSSIF-V2 \\
\hline Sodium Taurocholate $(\mathrm{mM})$ & 3 & 3 \\
\hline Lecithin $(\mathrm{mM})$ & 0.75 & 0.2 \\
\hline Dibasic sodium phosphate $(\mathrm{mM})$ & 28.65 & - \\
\hline Maleic acid $(\mathrm{mM})$ & - & 19.12 \\
\hline Sodium hydroxide $(\mathrm{mM})$ & 8.7 & 34.8 \\
\hline Sodium chloride $(\mathrm{mM})$ & 105.85 & 68.62 \\
\hline $\mathrm{pH}$ & 6.5 & 6.5 \\
\hline Osmolality $(\mathrm{mOsmol} / \mathrm{Kg})$ & $270 \pm 10$ & $180 \pm 10$ \\
\hline Buffer capacity $(\mathrm{mmol} / \mathrm{l} / \mathrm{pH})$ & 12 & 10 \\
\hline
\end{tabular}

Table 5: Composition of the media to simulate the Contents of the Small Intestine in the Fed State

\begin{tabular}{|c|c|c|c|c|c|}
\hline Contents of the Small Intestine & FeSSIF & Early & Middle & Late & FeSSIF-V2 \\
\hline Sodium Taurocholate $(\mathrm{mM})$ & 15 & 10 & 7.5 & 4.5 & 10 \\
\hline Lecithin $(\mathrm{mM})$ & 3.75 & 3 & 2 & 0.5 & 2 \\
\hline Glyceryl monooleate $(\mathrm{mM})$ & - & 6.5 & 5 & 1 & 5 \\
\hline Sodium oleate $(\mathrm{mM})$ & - & 40 & 30 & 0.8 & 0.8 \\
\hline Acetic acid & 140 & - & - & - & - \\
\hline Maleic acid & - & 28.6 & 44 & 58.09 & 55.02 \\
\hline Sodium hydroxide $(\mathrm{mM})$ & 101 & 52.5 & 65.3 & 72 & 81.65 \\
\hline Sodium chloride $(\mathrm{mM})$ & 173 & 145.2 & 122.8 & 51 & 125.5 \\
\hline pH & 5.0 & 6.5 & 5.8 & 5.4 & 5.8 \\
\hline Osmolality $(\mathrm{mOsmol} / \mathrm{Kg})$ & $635 \pm 10$ & $400 \pm 10$ & $390 \pm 10$ & $240 \pm 10$ & $390 \pm 10$ \\
\hline Buffer capacity $(\mathrm{mmol} / \mathrm{L} / \mathrm{pH})$ & 76 & 25 & 25 & 15 & 25 \\
\hline
\end{tabular}


Table 6: Composition of the Medium to Simulate the Colonic Fluid (SCoF)

\begin{tabular}{|c|c|}
\hline Composition & SCoF \\
\hline Acetic acid (mM) & 170 \\
\hline $\mathrm{NaOH}(\mathrm{mM})$ & 157 \\
\hline $\mathrm{pH}$ & 5.8 \\
\hline Osmolality (mOsmol/Kg) & 295 \\
\hline Buffer capacity $(\mathrm{mmol} / \mathrm{l} / \mathrm{pH})$ & 29.1 \\
\hline Ionic strength & 0.16 \\
\hline
\end{tabular}

$\mathrm{pH}$ decreases with increase in temperature and decreases when the temperature is lowered to $24^{\circ} \mathrm{C}$. The $\mathrm{pH}$ changes at elevated temperature necessitated addition of different amounts of $\mathrm{NaOH}$ for maintaining the medium within the physiological range. This could in turn have an influence on osmolality changes ${ }^{32}$. Osmolality is determined by freezing-point depression using an osmometer, buffer capacity is determined by titration with $1 \mathrm{M}$ hydrochloric acid and surface tension is measured using stalagnomet ${ }^{33}$. The dissolved gases can cause changes in the performance of the dissolution medium by changing $\mathrm{pH}$, forming bubbles on the dosage form or altering the interaction of the medium and the API. Extensive studies have been performed examining appropriate technique for the removal of dissolved gases from aqueous media which include a combination of heating and vaccum filtration followed by stirring under vaccum , vaccum filtration, heating, Sonication, helium sparing and membrane filtration ${ }^{34}$. For stability reason all media were used within $24 \mathrm{hrs}$ after preparation.

Taupitz et al suggested that these biorelevant media can be simplified by using SLS and Tween 80 to replace bile compounds ${ }^{35}$. In some cases of poorly water soluble drugs, existing media such as FaSSIF and FeSSIF were not enough to predict the absorption behavior of drug so that MREVID 1 and 2 was proposed as a new in-vitro dissolution medium to describe the in-vivo dissolution behavior ${ }^{36}$. The composition of MERVID dissolution medium shown in Table 7.

Table 7: Composition of medium reflecting in-vivo dissolution (MREVID)

\begin{tabular}{|l|l|l|}
\hline Composition & MREVID 1 & MREVID 2 \\
\hline Sodium Taurocholate $(\mathrm{mM})$ & 30 & 22.5 \\
\hline Phosphatidylcholine $(\mathrm{mM})$ & 7.5 & 5.625 \\
\hline Buffer $(\mathrm{g} / \mathrm{L})$ & & \\
KH2PO4 & 3.9 & 3.9 \\
KCL & 7.7 & 7.7 \\
\hline pH & 6.5 & 6.5 \\
\hline
\end{tabular}

Diakidou et al performed simulation of gastric lipolysis and predicted the Felodipine release from a matrix tablet in the fed stomach and concluded that modeling intragastric lipolysis is necessary in order to simulate felodipine release from extended release tablet in fed stomach $^{37}$.

\section{2) Hydrodynamics:}

The dissolution fluid flow characteristics should consist of a predictable pattern that is free of irregularities or variable turbulence. Hydrodynamics is predominant for (C) 2011, JDDT. All Rights Reserved the overall dissolution rate if the mass transfer process is mainly controlled by convection/diffusion as is usually the case for poorly soluble substances. A thorough knowledge of hydrodynamics is useful in the course of dissolution method development and formulation development for pharmaceutical industries quality needs 27. The dissolution apparatus used for different formulation are shown in Table 8 .

Table 8: Apparatus used for different formulation

\begin{tabular}{|l|l|}
\hline Types of dosage form & Release method \\
\hline Solid oral dosage form & $\begin{array}{l}\text { Basket,paddle,reciprocating } \\
\text { cylinder }\end{array}$ \\
\hline Implants & Modified flow through cell \\
\hline Chewing gum & Special apparatus \\
\hline Powders \& granules & Flow through cell \\
\hline
\end{tabular}

Muscular contraction in the wall of the small intestine achieve two objectives one is stirring of the contents to increase exposure to enzymes and to bring the luminaly digested products close to the wall and second propulsion of indigestible material towards the distal gut. Abrahamsson demonstrated that human intestinal hydrodynamics were reflected in vitro using the paddle method at stirring rates of about 140rpm. However, human studies to establish such correlation are expensive and time consuming. He had used, Labradors as the anatomy and physiology of GI tract of Labradors resembles those of the human GI tract. This canine breed can serve as a model to simulate human intestinal hydrodynamics. The pharmacokinetic of felodipine matrix tablet (poorly soluble, neutral and lipophilic) in Labrador was studied. On the other hand micronized and coarse felodipine dissolution was carried out in biorelevant medium at various speeds (slower, medium, fast). In vitro AUC ratio of this particular experimental set up showed best agreement with the pharmacokinetic parameters $^{27}$.

Cammarn et al have established a model for the dissolution of non-disintegrating salicylic acid tablets as a function of hydrodynamic conditions in the Flow Through Cell system (USP Apparatus 4). The approach was to model the dissolution rate of the material as a function of the Reynolds's number ${ }^{27}$. Reynolds number is commonly described as the ratio of momentum forces to viscous forces in a moving fluid ${ }^{38}$.

D'Arcy et al simulated hydrodynamics in the Levy beaker dissolution apparatus and explored the hydrodynamic characteristics of the apparatus. They also determined the hydrodynamics relevance to in vivo data used in IVIVCs through comparison of the magnitudes of velocities in the regions of the paddle, basket and Levy apparatuses where a dosage form would be located, with functions of in vitro dissolution rate data used in IVIVC studies ${ }^{39}$.

Gao Zongming compared dissolution testing under finite and infinite sink conditions, this study applied both paddle and flow-through methods for dissolution testing with disintegrating prednisone and nondisintegrating salicylic acid tablets. The closed- and open-loop 
configurations of the flow-through method were used to provide comparisons with the paddle method and the dissolution rates obtained using the two configurations were similar if the sink condition maintained ${ }^{40}$.

Mirza et al evaluated the dissolution Hydrodynamics in the USP, Peak ${ }^{\mathrm{TM}}$ and Flat-Bottom Vessels Using Different Solubility Drugs. The existence of the 'dead zone' at the bottom of the USP vessel was confirmed by performing perturbation (vessel tilt) studies using the USP Prednisone calibrator tablets, and two Novartis Development tablet formulations containing a low and a high solubility drug. All formulations formed a 'cone'of disintegrated mass at the bottom of the vessels. The hydrodynamic environment in the Peak ${ }^{\mathrm{TM}}$ and in flatbottom vessels was also evaluated using the low and high solubility drug formulations. There was no significant difference between the dissolution rates obtained by using the USP and flat-bottom vessels. The Peak vessel provided the highest release rates that were significantly different from those obtained by using USP and flatbottomed vessels. Finally, at higher paddle speeds of 60 and $75 \mathrm{rpm}$, the results obtained from USP vessels were comparable to results from the Peak vessel operated at 50 $\mathrm{rpm}^{41}$.

The NJIT group under Prof. Armenante studied the hydrodynamics of dissolution testing using LaserDoppler Velocimetry (LDV) and Computational Fluid Dynamics (CFD), respectively, to experimentally map and computationally predict the velocity distribution and the turbulent intensity inside a standard USP Apparatus II under the typical operating conditions mandated by the dissolution test procedure and concluded that the velocity in the region below the paddle is very low in magnitude 42

\section{3) API/Formulation characteristics}

Knowledge of the physiochemical nature of a compound in biorelevant media is useful for formulation development, which follows API phase selection. Based on this information the $\mathrm{pKa}$ profile of compound could be improved by modifying the surfactants or excipients in the formulation ${ }^{43}$. Many new chemical entities possess physiochemical characteristics unfavorable for oral absorption $^{44}$.

\section{BCS:}

A biopharmaceutical classification system is a scientific framework for classifying the drug substance based on their aqueous solubility and intestinal permeability. The BCS was first devised in 1995, by Amidon et al and since then it has become a benchmark in the regulation of bioequivalence of oral drug products. According to BCS classification drugs can be categorized as follows

Class 1: High solubility and high permeability

Class 2: Low solubility and high permeability

Class 3: High solubility and low permeability

Class 4: Low solubility and low permeability

For drugs belonging to class 1 and 3, simple aqueous media such as SGF and SIF (with or without enzymes) are suggested. In contrast for class 2 and 4 use of biorelevant media is recommended for dissolution testing. There are various methods of determination of solubility and permeability ${ }^{45}$. Galia et al 1998 showed use of biorelevant media to assess immediate release tablets. The study concludes that biorelevant media are preferable for BCS class 2 drugs, but do not improve the dissolution of BCS class 1 drugs.

\section{Solubility:}

Solubility is a crucial parameter for successful drug development as poor solubility compromises the Pharmacokinetic and Pharmacodynamic properties of drug (46). Solubility can be measured either thermodynamically or kinetically. Thermodynamic solubility can be defined as the concentration in solution of a compound in equilibrium with an excess of solid material at the end of the dissolution process and often considered as true solubility. Kinetic solubility considers the precipitation after dilution in a suitable solution of a compound predissolved in a co-solvent or in aqueous media by $\mathrm{pH}$ adjustment for ionizable compound ${ }^{47}$. Solubility of drug in biorelevant dissolution media increased compared to the solubility in aqueous buffer because of enhanced wetting and micellar Solubilization 48. Drug solubility testing in biorelevant media has become an indispensable tool in pharmaceutical development. Despite this importance, there is still an incomplete understanding of how poorly soluble compounds interact with these media. The study was carried out to apply the concept of the apparent solubilization capacity to fasted and fed state simulated intestinal fluid (FaSSIFand FeSSIF, respectively). A set of nonionized poorly soluble compounds was studied in biorelevant media prepared from an instantly dissolving complex at $37^{\circ} \mathrm{C}$.The values of the solubilisation capacity were different between FaSSIFand FeSSIF but correlated. Drug inclusion into the mixed micelles was highly specific for a given compound. The ratio of the FeSSIF to FaSSIF solubility was in particular considered and discussed in terms of the apparent solubilizing capacity. The apparent Solubilization concept appears to be useful for the interpretation of biorelevant solubility tests. Further studies are needed to explore acidic and basic drugs 49,50 .

\section{Particle Size:}

The dissolution rate is directly proportional to the surface area of the drug. Reducing particle size leads to an increase in the surface area exposed to the dissolution medium, resulting in a greater dissolution rate. Thus, the dissolution rate of poorly soluble drugs can often be enhanced markedly by undergoing size reduction (e.g., through micronization). However, particle size reduction does not always improve the dissolution rate. This is in part attributed to adsorption of air on the surface of hydrophobic drugs, which inhibits the wetting and hence reduces the effective surface area. In addition, fine particles tend to agglomerate in order to minimize the surface energy, which also leads to a decrease in the effective surface area for dissolution.

\section{Drug pKa and gastrointestinal pH: ${ }^{51}$}


The amount of drug that exists in unionized form is a function of dissociation constant (pKa) of the drug and $\mathrm{pH}$ of the fluid at absorption sites. The relation between drug $\mathrm{pKa}$ and ionization and absorption is shown in Table 9.

Table 9: Relation between drug pKa and ionization

\begin{tabular}{|l|l|l|}
\hline Sr.No & pKa range \& drug nature & $\mathrm{pH} /$ site of absorption \\
\hline 1) & Stronger acid(pKa $<2.5)$ & Ionized at all $\mathrm{pH}$ values; Poorly absorbed from GIT. \\
\hline 2) & $\begin{array}{l}\text { Moderately weak acid }(\mathrm{pKa}=2.5- \\
\text { 7.5) }\end{array}$ & $\begin{array}{l}\text { Unionized in gastric } \mathrm{pH} \text { values and ionized in intestinal; better } \\
\text { absorbed from stomach. }\end{array}$ \\
\hline 3$)$ & Very weak acid(pKa $>8)$ & Unionized at all $\mathrm{pH}$ values; absorbed along the entire length of GIT. \\
\hline 4$)$ & Stronger base(pKa $>11)$ & Ionized all $\mathrm{pH}$ values; Poorly absorbed from GIT. \\
\hline 5$)$ & Moderately weak base $(\mathrm{pKa}=5-11)$ & $\begin{array}{l}\text { Ionized at gastric } \mathrm{pH} \text {; relatively unionized at intestinal } \mathrm{pH} \text {; better } \\
\text { absorbed from intestine. }\end{array}$ \\
\hline
\end{tabular}

\section{4) Prediction of plasma profile}

In vitro drug dissolution/release tests are conducted to estimate or predict in vivo drug release characteristics of a product. Direct estimation of in vivo drug dissolution is usually not possible and therefore blood drug concentration-time profiles are used for this purpose ${ }^{52}$. The prediction of plasma profile can be done by using model dependent or model independent approaches. Wagner-Nelson, Loo-Riegelman and numerical deconvolution are such methods. Wagner-Nelson and Loo-Riegelman are both model dependent methods in which former is used for a one-compartment model and the latter for multi-compartment system ${ }^{53}$. The prediction method using convolution analysis consists of following processes ${ }^{54}$.

1) The drug amount-time profile in each segment is calculated by the convolution method.

2) The absorption rate-time profile in each segment is calculated by using the drug amount-time profile in each segment, calculated in step 1 .

3) The absorption rate-time profile in the whole GI tract is calculated as the sum of the absorption rate-time profiles of four segments obtained in step 2 .

4) Prediction of the plasma concentration-time curve of orally administered drug is performed by means of the convolution method.

5) The total absorption rate- time data obtained in step 3 and pharmacokinetic parameters after intravenous administration correspond to the input function and the weight function, respectively. The inverse Laplace transformation of the obtained equation by the convolution program gives the predicted plasma concentration profile after oral administration without the first-pass metabolism in intestinal epithelium and/or liver.

The pharmacokinetic parameters determined by taking following consideration as study design, population study, study conditions, characteristics investigated during bioavailability study, bioanalytical methodology and statistical evaluation ${ }^{55}$.

Rahman et al derived absorption profiles of Theophylline by using Wagner-Nelson equation.

Fujioka et al tried to predict the in-vivo absorption kinetics of griseofulvin orally administered as a powder into rats, based on gastrointestinal transit absorption model (GITA), consisting of absorption, dissolution and GI- transit processes ${ }^{56}$.

\section{5) Development of IVIVCs}

The term correlation is frequently employed within the pharmaceutical and related sciences to describe the relationship that exists between variables. Mathematically, the term correlation means interdependence between quantitative or qualitative data or relationship between measurable variables and ranks. From biopharmaceutical standpoint, correlation could be referred to as the relationship between appropriate in vitro release characteristics and in vivo bioavailability parameters. Two definitions of IVIVC have been proposed by the USP and by the FDA.

\section{United State Pharmacopoeia (USP) definition}

The establishment of a rational relationship between a biological property and a parameter derived from a biological property produced by a dosage form, and a physicochemical property or characteristic of the same dosage form.

\section{Food and Drug Administration (FDA) definition}

IVIVC is a predictive mathematical model that shows relationship between an in vitro property of a dosage form and a relevant in vivo response. Generally, the in vitro property is the rate or extent of drug dissolution or release while the in vivo response is the plasma drug concentration or amount of drug absorbed.

\section{OBJECTIVES OF IVIVC ${ }^{57,58}$}

IVIVC plays an important role in product development which serves as a surrogate of in vivo and assists in supporting biowaivers, supports and / or validates the use of dissolution methods and specifications and assists in quality control during manufacturing and selecting appropriate formulations. The different levels of IVIVC are listed in Table $10^{59,60}$.

To develop and validate an IVIVC model, two or three different formulations should be studied in vitro and in vivo (FDA guidance, 1997). Typically, the qualitative composition of drug products is the same, but the release-controlling variable(s), e.g., the amount of excipients, or a property of the drug substance such as 
particle size, is varied. To develop a discriminative in vitro dissolution method, several method variables together with formulation variables are studied, e.g., different $\mathrm{pH}$ values, dissolution apparatuses and agitation speeds

Table 10: Levels of IVIVCs

\begin{tabular}{|l|l|l|}
\hline Levels & In vitro & In vivo \\
\hline $\mathrm{A}$ & Dissolution curve & Absorption curve \\
\hline $\mathrm{B}$ & Statistical moment MDT & Statistical moments MRT,MAT etc. \\
\hline $\mathrm{C}$ & $\begin{array}{l}\text { Disintegration time, time to have 10, 50, 90\% } \\
\text { dissolved, dissolution rate, dissolution efficiency }\end{array}$ & $\begin{array}{l}\text { Cmax, Tmax, Ka time to have 10, 50, 90 } \\
\% \text { absorbed, AUC }\end{array}$ \\
\hline Multiple level C & $\begin{array}{l}\text { One or several pharmacokinetic parameters of } \\
\text { interest }\end{array}$ & $\begin{array}{l}\text { Amount of drug dissolved at several time } \\
\text { points }\end{array}$ \\
\hline $\mathrm{D}$ & Not considered useful for regulatory purpose & \multicolumn{2}{|l|}{} \\
\hline
\end{tabular}

Mainly two approaches are used for development of correlation

\section{* Two step}

Step 1: Estimate the in vivo absorption or dissolution time course using an appropriate technique for each formulation and subject

Step 2: establish link model between in vivo and in vitro variables and predict plasma concentration from in vitro using the link model.

\section{* One step}

Predict plasma concentration from in vitro using a link model whose parameters are fitted in one step, so here it doesn't involve deconvolution.

The deconvolution technique requires the comparison of in vivo dissolution profile obtained from the blood profiles with in vitro dissolution profiles. It is the most commonly cited and used method in the literature. Perhaps that is the reason for the lack of success of developing IVIVC, since this approach is conceptually weak and difficult to use to derive the necessary parameters for their proper evaluation. For example: (1) Extracting in vivo dissolution data from a blood profile often requires elaborate mathematical and computing expertise. (2) It often requires multiple products having potentially different in vivo release characteristics (slow, medium, fast). These products are then used to define experimental conditions (medium, apparatus etc.) for an appropriate dissolution test to reflect their in vivo behaviour. (3) This technique requires blood data (human study) for the test products to relate it to in vitro results.

An IVIVC should be evaluated to demonstrate that predictability of in vivo performance of a drug product from its in vitro dissolution characteristics is maintained over a range of in vitro dissolution release rates and manufacturing changes. Since the objective of developing an IVIVC is to establish a predictive mathematical model describing the relationship between an in vitro property and a relevant in vivo response, the proposed evaluation approaches focus on the estimation of predictive performance or, conversely, prediction error. Methodology for the evaluation of IVIVC predictability is an active area of investigation and a variety of methods are possible and potentially acceptable. A correlation should predict in vivo performance accurately and consistently.

Internal predictability is applied to IVIVC established using formulations with three or more release rates for non-narrow therapeutic index drugs exhibiting conclusive prediction error ${ }^{62}$.

$$
\% \mathrm{PE}=[(\text { Observed parameter }- \text { Predicted parameter }) /
$$

According to the IVIVC guidance, the average prediction error across formulations cannot be greater than $10 \%$ and a formulation cannot have a prediction error greater than $15 \%$. Based on these criteria, each of the IVIVC models is valid in terms of the rate and extent of drug absorption 63.

External predictability evaluation is not necessary unless the drug is a narrow therapeutic index, or only two release rates were used to develop the IVIVC, or, if the internal predictability criteria are not met i.e. prediction error internally is inconclusive. However, since the IVIVC will potentially be used to predict the in vivo performance for future changes, it is of value to evaluate external predictability when additional data are available 64 .

The prediction error for external validation should not exceed $10 \%$ where as \% PE between $10-20 \%$ indicates inconclusive predictability and the need for further study using additional data sets.

Various softwares have been developed such as Simcyp, GastroPlus $^{\mathrm{TM}}$, PK-Sim ${ }^{\mathrm{TM}}$, MEDICI-PK ${ }^{\mathrm{TM}}$, Cloe $\mathrm{PK}^{\mathrm{TM}}$ etc. for physiological based pharmacokinetic model $(\mathrm{PBPK})^{65,66}$.

The parameter such as metabolic factors, drug loss in GIT and stereochemistry are to be considered while developing IVIVC ${ }^{67}$.

Souliman et al. compared two in vitro models using a class I substance and found that the best IVIVC existed using an artificial digestive system. Thus, development of improved IVIVCs is possible using various models and fluids meant to simulate physiological conditions ${ }^{68}$, 69. 


\section{CONCLUSION}

The development of Biorelevant dissolution medium mainly used as in vitro surrogate for in vivo performance. The compendial dissolution medium is unable to simulate the dissolution as that of in vivo so

\section{REFERENCES}

1. He Z, Zhong D, Chen X, Liu X, Tang X, Zhao L. Development of dissolution medium for nimodipine tablets based on bioavailability evaluation. Eur $\mathrm{J}$ of Pharma Sci. 2004; 21: 487-491.

2. Gander B, Ventouras K, Gurny R, Doelkar E. In vitro dissolution medium with supramicellar surfactant concentration and its relevance for in vivo absorption. Int $\mathrm{J}$ of Pharma. 1985; 27: 117-124.

3. Lehto P. Academic Dissertation; Division of Pharmaceutical Technology Faculty of Pharmacy University of Helsinki Mechanistic Studies of Drug Dissolution Testing Implications of solid phase properties and in vivo prognostic media; Academic Dissertation 2010; 1-49.

4. Yang L. Biorelevant dissolution testing of colon-specific delivery systems activated by colonic microfora. $\mathrm{J}$ of controlled Release. 2008; 125: 77-86.

5. Dickinson P, Lee W, Stott P, Townsend A, Smart J, Ghahramani P, Hammett T, Billett L, Behn S, Gibb R, Abrahamsson B. Clinical Relevance of Dissolution Testing in Quality by Design. AAPS PharmSciTech. 2008: 10(2)

6. Lakshmana $\mathrm{P}$, Singh $\mathrm{T}$, Dinesh $\mathrm{K}$. Development of dissolution medium for poorly water soluble drug Racecadotril. J Pharm Research. 2009; 4: 213-215.

7. Maggi L, Torre M, Giunchedi P, Conte U. Supramicellar solutions of sodium dodecyl sulphate as dissolution media to study the in vitro release characteristics of sustained-release formulations containing an insoluble drug: nifedipine; Int $\mathrm{J}$ of Pharm. 1996; 135: 73-79.

8. Jamzad S, Fassihi R. Role of Surfactant and $\mathrm{pH}$ on Dissolution Properties of Fenofibrate and Glipizide-A Technical Note. AAPS PharmSciTech. 2006; 7 (2): E1-E6.

9. El-massik M, Darwish I, Hassan E, El-Khordagui L. Development of dissolution medium for gliblenclamide. Int $\mathbf{J}$ of Pharm. 1996; 140: 69-76.

10. Grove M, Mullertz A, Pedersen G, Nielsen J. Bioavailability of Seocalcitol Administration of lipid-based formulations to minipigs in the fasted and fed state. Eur J of Pharm Sci. 2007; 31: 8-15

11. Marques M. Dissolution Media Simulating Fasted and Fed States; Dissolution Technol. 2004; 11(2):16.

12. Klein S. The Use of Biorelevant Dissolution Media to Forecast the In Vivo Performance of a Drug. AAPS PharmaSciTech. 2010; 12(3): 397-406.

13. Sunesen V, Pedersen B, Kristensen H, Müllertz A. In vivo in vitro correlations for a poorly soluble drug, danazol, using the flow-through dissolution method with biorelevant dissolution media. Eur J Pharm Sci. 2005; 24: 305-313.

14. Kloefer B, Hoogevest P. Technical Notes: Using Biorelevant Media with Different Types of Orally Administered Formulations. Dissolution Technol. 2010; 17(2): 46-47.

15. Jantratid E, Dressman J. Biorelevant Dissolution media simulating conditions in the proximal human gastrointestinal tract: an update. Dissolution Technol. 2009; 16(3): 21-25.

16. Single N, Gupta G, Kohali K, Singla A. A Discriminative and Biorelevant Dissolution Test Method for Simvastatin Drug Products. Dissolution Technol. 2009; 16(4): 11-13.

17. Wei H, Lobenberg R. Biorelevant dissolution media as a predictive tool for glyburide a class II drug. Eur J Pharm Sci. 2006; 29 (1): 45-52.

18. Zoeller $\mathrm{T}$, Klein $\mathrm{S}$. Simplified Biorelevant Media for Screening Dissolution Performance of Poorly Soluble Drug. Dissolution Technol. 2007; 14(4): 8-13.

19. Juenemann D, Jantratid E, Wagner C, Reppas C, Vertzoni M, Dressman J. B. Biorelevant in vitro dissolution testing of that the development of Biorelevant dissolution medium is necessary. During the development of Biorelevant medium the necessary steps should be consider which can be applied to assess drugs and their dosage forms during the course of drug product development.

products containing micronized or nanosized fenofibrate with a view to predicting plasma profiles. Eur $\mathbf{J}$ of Pharma and Biopharmaceutics; 2011; 77: 257-264.

20. Jantratid E, De Maio V, Ronda E, Mattavelli V, Vertzoni M, Dressman, J. Application of biorelevant dissolution tests to the prediction of in vivo performance of diclofenac sodium from an oral modified-release pellet dosage form. Eur $\mathrm{J}$ Pharm Sci. 2009; 37: 434-441.

21. Wang Q, Fotaki N, Mao Y. Biorelevant Dissolution: Methodology and Application in Drug Development. Dissolution Technol. 2009; 16 (3): 6-12.

22. Sheng J, Kasim N.A, Chandrasekharan R, Amidon G. Solubilization and dissolution of insoluble weak acid, ketoprofen: Effects of $\mathrm{pH}$ combined with surfactant. Eur $\mathrm{J}$ of Pharm Sci. 2006; 29: 306-314.

23. Di Maio S, Carrier R. Gastrointestinal contents in fasted state and post-lipid ingestion: in vivo measurements and in vitro models for studying oral drug delivery. $\mathrm{J}$ of Controlled release; 2010.

24. Al-Behaisi S.K. In vitro modeling of food effect influencing dissolution of acid-labile drugs; Ph.D. Thesis; Doctoral school of pharmaceutical and pharmacological sciences; 2002;1-10.

25. Willmann S, Thelen K, Becker C, Dressman J, Lippert J. Mechanism-based prediction of particle size-dependent dissolution and absorption: Cilostazol pharmacokinetics in dogs Eur J of Pharm and Biopharmaceutics. 2010; 76: 83-94.

26. Peddireddy M.K.R. Pharmacological tools for the correction of GI motility disorders; Ind J Pharm Edu Research. 2011; 45(1): 15-24

27. Dressman J, Kramer J. Pharmaceutical dissolution testing; 2005.

28. Ghazal H, Dyas A, Ford J, Hutcheon G. In vitro evaluation of the dissolution behaviour of itraconazole in biorelevant media; Int J of pharm. 2009; 366: 117-123.

29. Jantratid E, Janssen N, Chokshi H, Tang K, Dressman J. Designing biorelevant dissolution tests for lipid formulations: case example--lipid suspension of RZ-50. Eur J Pharm Biopharm. 2008; 69: 776-785.

30. Vertzoni M, Dressman J, Butler J, Hempenstall J, Repass C. Simulation of fasting gastric acid conditions and its importance for the in vivo dissolution of lipophilic compounds. Eur J of Pharm and Biopharmaceutics. 2005; 60(9): 413-417.

31. Fotaki N, Vertzoni M. Biorelevant dissolution methods and their applications in In vitro-in vivo correlations for oral formulations.

32. Iyer S, Barr $\mathrm{W}$, Karnes $\mathrm{H}$. Characterization of a potential medium for 'biorelevant' in vitro release testing of a naltrexone implant, employing a validated stability-indicating HPLC method. J of Pharm and Biomedical Analysis. 2007; 43 845-853.

33. Jogia H, Mehta T, Patel M. Evaluation of dissolution media a novel synthetic surfactant by in vitro testing of BCS class 2 drugs. Dissolution Technol. 2009; 16(3): 14-19.

34. Fliszar K, Forsyth R, Li Z, Martine G. Effects of dissolved gases in surfactant dissolution media. Dissolution Technol. 2005; 12(3): 6-9.

35. Taupitz T, Klein S. Biorelevant media be simplified by using SLS and Tween 80 to Replace Bile Compounds? The open Drug Deliv J. 2010; 4: 30-37.

36. Fujioka Y, Kadono K, Fujie Y, Metsugi y, Ogawara K, Higaki K, Kimura T. Prediction of oral absorption of griseofulvin, a BCS class 2 drug, based on GITA model; 
Utilization of a more suitable medium for in-vitro dissolution study. J of Controlled release. 2007; 119: 222-228.

37. Diakidou A, Vertzoni M, Abrahamsson B, Dressman J, Reppas C. Simulation of gastric lipolysis and prediction of felodipine release from a matrix tablet in the fed stomach. Eur J of Pharm Sci. 2009; 37: 133-140.

38. Cammarn S, Sakr A. Predicting dissolution via hydrodynamics: salicylic acid tablets in flow through cell dissolution. Int J of Pharm. 2000; 201: 199-209.

39. D'Arcy D, Healy A, Corrigan O. Towards determining appropriate hydrodynamic conditions for in vitro in vivo correlations using computational fluid dynamics. Eur $\mathbf{J}$ of Pharm Sci. 2009; 37:291-299.

40. Gao Z. In Vitro Dissolution Testing with Flow-Through Method: A Technical Note; AAPS PharmSciTech. 2009; 10(4): 1401-1405.

41. Mirza T, Joshi Y, Liu Q, Vivilecchia R. Evaluation of Dissolution Hydrodynamics in the USP, Peak ${ }^{\mathrm{TM}}$ and FlatBottom Vessels Using Different Solubility Drugs. Dissolution Technol. 2005; 12(1): 11-16.

42. Armenante P. Inherent Method Variability in Dissolution Testing: The Effect of Hydrodynamics in the USP II Apparatus; 1-21.

43. Juenemann $\mathrm{D}$, Bohets $\mathrm{H}$, Ozdemir $\mathrm{M}$, Maesschalk $\mathrm{r}$, Vanhoutte K, Peters K, Nagels L, Dressman J. Online monitoring of dissolution tests using dedicated potentiometric sensors in biorelevant media. Eur J of Pharm Sci. 2011; 1-8.

44. Azarmi S, Roa W, Lobenberg R. Current perspectives in dissolution testing of conventional and novel dosage forms. Int J of Pharma. 2007; 328: 12-21.

45. Sachan N, Bhattacharya A, Pushkar S, Mishra A. Biopharmaceutical classification system: a strategic tool for oral drug delivery technology. Asian J of pharm. 2009; 76-81.

46. Furukawa S, Zhao C, Ohki Y. Methodology for phase selection of a weak basic drug candidate, utilizing kinetic solubility profiles in biorelevant media. Eur $\mathrm{J}$ of Pharm and Biopharmaceutics. 2010; 74; 298-303.

47. Bard B, Martel S, Carrupt P. High throughput UV method for the estimation of thermodynamic solubility and the determination of the solubility in biorelevant media. Eur $\mathrm{J}$ of Pharm Sci. 2008; 33: 230-240.

48. Ottaviani G, Gosling D.J, Patissier C, Rodde S, Zhou L, Faller B. What is modulating solubility in simulated intestinal fluids? Eur Journal of Pharm Sci. 2010; 41: 452-457.

49. Darwish I, Elimassik M, Hassan E, El-Khordagui L. Assesment of a hydroalcoholic surfactant solution as a medium for the dissolution testing of phenytoin. Int $\mathrm{J}$ of pharm. 1996; 140: 25-32.

50. Schwebel H, Hoogevest P, Leigh M, Kuentz M. The apparent solubilizing capacity of simulated intestinal fluids for poorly water-soluble drugs; Pharm Dev and Techno; 2010; 1-9.

51. Brahmankar D, Jaiswal S. Biopharmaceutics and pharmacokinetics: A Treatise. Reprint edition 2007; Vallabh prakashan: 34-35.

52. Qureshi S. Determining blood concentration-time (C-t) profiles from in vitro dissolution results and product evaluation-carbamazepine; 1-5

53. Yasir M, Asif M. Ammeduzafar, Chauhan I, Singh A. In Vitro - In Vivo Correlation: A Review. Drug Invention Today. 2010; 2(6): 282-286.

\section{ABBREVATIONS:}

$\begin{array}{ll}\text { API } & \text { Active Pharmaceutical Ingradient } \\ \text { AUC } & \text { Area under curve } \\ \text { BCS } & \text { Biopharmaceutical classification system } \\ \text { CFD } & \text { Computational Fluid Dynamics } \\ \text { FDA } & \text { Food and drug administration } \\ \text { FeSSGF } & \text { Fed state simulated gastric fluid } \\ \text { FeSSIF } & \text { Fed state simulated intestinal fluid } \\ \text { FaSSIF } & \text { Fasted state simulated intestinal fluid }\end{array}$

54. Haruta S, Kawai K, Nishii R, Jinnouchi S, Ogawara K, Higaki K, Tamura S, Arimori K, Kimura T. Prediction of plasma concentration-time curve of orally administered theophylline based on a scientigraphic monitoring of gastrointestinal transit in human volunteers.; Int J of Pharm. 2002; 233: 179-190.

55. Guidance for Industry Immediate Release Solid Oral Dosage Forms Scale-Up and Postapproval Changes: Chemistry, Manufacturing, and Controls, In Vitro Dissolution Testing, and In Vivo Bioequivalence Documentation Center for Drug Evaluation and Research (CDER) November 1995 CMC 5.

56. Rahman N, Tulain U. Comparative bioavailability and in vitro in vivo correlation of two sustained release brands of theophylline: tablets and pellets. Pak J Pharm Sci. 2008; 21(2): 131-138.

57. Qureshi S; In Vitro-In Vivo Correlation (IVIVC) and Determining Drug Concentrations in Blood from Dissolution Testing-A Simple and Practical Approach. The Open Drug Deliv J. 2010; 4: 38-47.

58. Sirisuth N, Erdington N. In-Vitro-In-Vivo Correlation Definitions and Regulatory Guidance. Int $\mathrm{J}$ of Generic Drugs.2002; Part 2: 1-11.

59. Cardot J, Beyssac E, Alric M. In Vitro-In Vivo Correlation: Importance of Dissolution in IVIVC. Dissolution Technol. 2007; 15-19.

60. Yadav A, Kalaskar S, Patil V. In vitro-in vivo correlation: A ground discussion. Ind J Pharm.Edu. Res. 2007; 41(4): 306318.

61. Kortejarvi H. Modelling and simulation approaches for waiving in vivo pharmacokinetic formulation studies; Division of Biopharmaceutics and Pharmacokinetics Faculty of Pharmacy university of Helsinki Finland; Academic Dissertation; 2008; 1-65.

62. Emami J. In vitro-in vivo correlation: from theory to applications. J Pharm Sci. 2006; 9(2):169-189.

63. Jacobs T, Rossenu S, Dunne A, Molenberghs G, Straetemans R, Bijnens L. Combined Models for Data From In Vitro-In Vivo Correlation Experiments. 1-26.

64. Eddington N. In Vitro In Vivo Correlation with Metoprolol Extended Release Tablets Using Two Different Releasing Formulations: An Internal Validation Evaluation. Int $\mathrm{J}$ of Generic Drugs. 417-429.

65. Lave T, Parrott N, Grimm H, Fleury A, Reddy M. Challenges and opportunities with modelling and simulation in drug discovery and drug development Xenobiotica. 2007; 37(10): $1295-1310$.

66. Okumua A, DiMaso M, Lobenberg R. Computer simulations using GastroPlusTM to justify a biowaiver for etoricoxib solid oral drug products. Eur $\mathbf{J}$ of Pharma and Biopharmaceutics. 2009; 72: 91-98.

67. Ghosh A, Choudhury G. In vitro-In vivo Correlation (IVIVC): A Review. J of Pharma Research. 2009; 2(8): 12551260.

68. Souliman S, Blanquet S, Beyssac E, Cardot J. A level A in vitro invivo correlation in fasted and fed states using different methods; applied to solid immediate release oral dosage form. Eur J of pharma sci. 2006; 27(1): 72-79.

69. Lue B, Nielsen F, Magnussen T, Schou H, Kristensen K, Jacobsen L, Müllertz A. Using biorelevant dissolution to obtain IVIVC of solid dosage forms containing a poorlysoluble model compound. Eur. J. Pharm. Biopharm. 2008; 69: 648-657.

$\begin{array}{ll}\text { GIT } & \text { Gastrointestinal tract } \\ \text { GITA } & \text { Gastrointestinal transit absorption model } \\ \text { GMO } & \text { Glycerylmonooleate } \\ \text { IVIVC } & \text { In vitro in vivo correlation } \\ \text { LDV } & \text { Laser-Doppler Velocimetry } \\ \text { MERVID } & \text { Medium reflecting in-vivo dissolution } \\ \text { NJIT } & \text { New jersey institute of technology } \\ \text { USP } & \text { United state of pharmacopoeia }\end{array}$

